\title{
ACTITUDES, CONOCIMIENTOS, MITOS Y FALSAS CREENCIAS DE LOS ADOLESCENTES ANTE LA VIOLENCIA DE GÉNERO
}

\author{
María Abad Jambrina \\ Educadora Social \\ maria.abadjambrina@gmail.com \\ Ana Isabel Isidro de Pedro \\ Profesora Titular de E.U. \\ Departamento de Psicología Social y Antropología. \\ Universidad de Salamanca \\ anyis@usal.es
}

Fecha de Recepción: 26 Marzo 2018

Fecha de Admisión: 10 Abril 2018

\section{RESUMEN}

En el presente trabajo se analizan los conocimientos, actitudes, pensamientos -mitos y falsas creencias- y comportamientos de una muestra de jóvenes ante la violencia de género en las relaciones de pareja. El estudio se basa en analizar y comparar dos grupos de adolescentes. Por un lado, estudiantes pertenecientes a centros educativos normalizados y, por otro, jóvenes considerados en riesgo de exclusión social, ya que se encuentran en un centro de menores. Se pretende comprobar si existen diferencias entre ellos y de qué manera pueden influir en estos últimos los factores de riesgo a los que se encuentran expuestos. Concretamente, se han estudiado sus actitudes -de tolerancia y/o rechazo-, comportamientos, el grado de asimilación de los mitos y falsas creencias al respecto, así como la consciencia de los jóvenes al realizar diferentes prácticas en las redes sociales y ante la importancia de los medios de comunicación a la hora de interiorizar roles de género. A tal efecto, se utilizó un cuestionario de elaboración propia de 28 ítems. La muestra estuvo conformada por 119 sujetos con edades comprendidas entre los 13 y los 18 años. De los cuales, 61 adolescentes $(49,18 \%$ chicas y $50,82 \%$ chicos $)$-con una media de edad de 15,5 años- pertenecían a centros educativos denominados normalizados y 58 ( $51,72 \%$ chicas y $48,28 \%$ chicos) pertenecían a un centro de menores y tenían una media de edad de 15,8 años. Tras analizar, agrupar, contrastar e interpretar los datos, y según los resultados obtenidos, constatamos que la importante cantidad de proyectos implementados para luchar contra la violencia de género no son suficientes o no son plenamente eficaces, ya que ambos grupos de adolescentes estudiados no parecen tener los conocimientos suficientes como para cambiar su percepción y su modo de actuar ante dicha lacra.

Palabras clave: violencia de género; adolescentes; educación; sociedad 


\section{ABSTRACT}

Attitudes, knowledge, myths and false beliefs of adolescents faced with of gender violence.

In the present work, knowledge, attitudes, thoughts -myths and false beliefs- and behaviors for a sample of young people faced with gender violence into the couple relationships are analyzed. The study is based on analyzing and comparing two groups of adolescents. On the one hand, students belonging to standardized educational centers and, on the other, young people considered at risk of social exclusion, since they are in a juvenile reclusion center. We want to check if there are differences between them and how the risk factors to which this last group is exposed can influence on them.

Specifically, we have studied their attitudes - of tolerance and/or refusal-, behaviors, the degree of assimilation of both the myths and the false beliefs, as well as the awareness of young people both when performing different practices in the social networks and considering the importance of the media when internalizing gender roles. For this purpose, a questionnaire of 28 items, developed by the authors, was used. The sample consisted of 119 individuals aged between 13 and 18 years. Of which, 61 adolescents (49,18\% girls and $50,82 \%$ boys) with an average age of 15,5 year belonged to standardized educational centers and 58 (51,72\% girls and $48,28 \%$ boys) belonged to a juvenile reclusion center, having an average age of 15,8 year. After analyzing, grouping, contrasting and interpreting the data, and based on the results obtained, we conclude that the significant number of projects implemented to fight gender violence are either not enough or not fully effective, since both groups of adolescents studied do not seem to have enough knowledge to change their perception and their way of acting in the face of this scourge.

Keywords: gender violence; adolescents; education

\section{ANTECEDENTES}

La Organización de las Naciones Unidas (ONU) define la violencia de género como cualquier tipo de violencia contra sexo femenino que tenga o pueda tener como resultado daño o sufrimiento físico, sexual o psicológico para la mujer, así como las amenazas de tales actos, la coacción o la privación arbitraria de la libertad, tanto en la vida pública como en la vida privada (ONU, 1994). Es una de las definiciones más reconocidas y posee un carácter internacional.

Por otra parte, la Organización Mundial de la Salud (2017) sitúa la adolescencia como un periodo comprendido entre los 10 y los 19 años de edad. Consta de dos etapas, la primera, entre los 10 y los 14 años, y la segunda entre los 15 y los 19. Ésta última es una etapa de cambios rápidos y profundos a nivel físico, psicológico y social del ser humano y en ella suelen iniciarse las primeras relaciones de pareja y noviazgos.

Una de las formas sexistas de construir el autoconcepto de los adolescentes es a través de los estereotipos, los cuales se aprenden, asientan y trasfieren de forma social y cultural. A través del aprendizaje por modelado, aprenden de lo que observan de los otros. Éste es un proceso de aprendizaje en el que intervienen, además de los diferentes factores culturales comunes en la sociedad, otros como la influencia de la familia y la escuela. Los estudios sobre la adquisición de los estereotipos de género refieren que el sexo es una clave utilizada muy pronto por los niños y niñas y que con un conocimiento muy elemental de género se reproducen ya conductas estereotipadas. Además, según va aumentando la edad, la red de asociaciones respecto al género es cada vez más elaborada y la relación que se obtiene entre las características de lo femenino y lo masculino se va configurando como divergente (Williams y Best, 1990).

Por su parte, González y Santana (2001) detectan la gran influencia que ejercen sobre los jóvenes los mitos relacionados con el amor romántico. Éstos ayudan a mantener en ellos una visión excesivamente romántica del amor, pudiéndose producir sentimientos de tolerancia ante una situa- 
ción donde el amor se utiliza como justificación del control que la pareja puede ejercer sobre el otro. Esta visión contribuye a la relación que los adolescentes pueden tener entre masculinidad y control.

Acompañando a lo anterior, les suele dar vergüenza hablar sobre los temas relacionados con su intimidad. Por ello, muchas veces tienen miedo a revelar delante de los adultos la situación de violencia que están sufriendo. Tienen miedo a la reacción de éstos, a que subestimen lo que les está pasando, a que los controlen, a que denuncien a su pareja, a que se conozca la situación o a que de ello se derive una eliminación o merma de la autonomía que tenía sobre su propia vida. Por todo esto, intentan solucionar los problemas por su cuenta, ocultando la situación (Cantera, Estébanez y Vázquez, 2009).

Después de lo anterior, debemos ser conscientes de que la violencia se suele instalar en las relaciones de forma gradual y que en muchas ocasiones no se manifiesta hasta que no se comienza la convivencia.

\section{OBJETIVOS}

Adquirir información sobre la percepción y conocimiento que tienen los adolescentes sobre la violencia de género.

Comprobar si los adolescentes conocen adecuadamente el concepto de violencia de género, así como cuáles son las diferentes formas en las que se puede manifestar dentro de una pareja.

Conocer cuáles son los mitos y falsas creencias que los adolescentes tienen interiorizados como verdaderos y que utilizan a la hora de buscar pareja.

Analizar las actitudes tolerantes y de rechazo que tienen los adolescentes ante una situación de violencia de género.

Averiguar la consciencia de los adolescentes a la hora de realizar diferentes prácticas en las redes sociales y la importancia de los medios de comunicación a la hora de interiorizar roles de género.

Saber si existen diferencias a la hora de percibir la violencia de género según diferentes variables.

Saber si existen diferencias significativas en el aprendizaje de roles y estereotipos de género según las dificultades del menor en su experiencia vital.

Conocer si existen diferencias importantes en el conocimiento acerca de la definición de violencia de género y de los diferentes tipos que podemos encontrar en las relaciones.

Comprobar si existen grandes diferencias entre las actitudes que los adolescentes adptan sobre los comportamientos violentos en pareja.

Conocer si existen diferencias a la hora de interiorizar mitos y falsas creencias según en centro donde se encuentren.

Saber si existen diferencias a la hora de utilizar las nuevas tecnologías.

\section{METODOLOGÍA}

La investigación realizada sigue un método descriptivo que se ha basado en la aplicación de un cuestionario de elaboración propia integrado por 28 ítems (referidos a tres grandes bloques que posteriormente se especificarán), con el fin de conocer las diferentes percepciones, actitudes y comportamientos que tienen los adolescentes en relación con la violencia de género, así como la interiorización de estereotipos, mitos y falsas creencias aprendidos desde la infancia.

De este modo, se ha querido indagar acerca del conocimiento que tienen los jóvenes sobre del concepto de violencia de género y si es un concepto del que tienen clara la definición y los diferentes tipos que existen. Igualmente, se ha examinado la influencia que ejercen los medios de comunicación y las nuevas tecnologías sobre los adolescentes y si son conscientes de la importancia que 
tienen aquéllos a la hora de proporcionarles roles y estereotipos de género, comprobando también si realizan un uso adecuado y consciente de las nuevas tecnologías.

Además, el estudio también se ha realizado de forma comparativa, para observar si existen diferencias entre adolescentes en riesgo de exclusión social (residentes en un centro de menores) y adolescentes que, a priori, no presentan tal riesgo, residen con sus familias y estudian en centros normalizados. Esto nos servirá para contrastar los conocimientos que ambos grupos tienen acerca de la violencia de género, la formación recibida sobre este ámbito y si han tenido alguna experiencia cercana relacionada con este problema.

Una vez recogidos los datos, éstos se han tratado mediante el paquete estadístico de software libre PSPP y, posteriormente, se ha usado Microsoft Excel para realizar las figuras.

Por lo que a la muestra se refiere, está constituida por 119 adolescentes, con edades comprendidas entre los 13 y los 18 años. De ellos, 58 están en riesgo de exclusión y pertenecen a un centro abierto de menores ( $\bar{x}=15,8$ años) y 61 no presentan tal riesgo y cursan estudios en diferentes centros normalizados ( $\bar{x}=15,5$ años).

\section{RESULTADOS}

Han respondido al cuestionario, dentro del grupo de adolescentes pertenecientes a los centros normalizados (ver Figura 1), un $49,18 \%$ de mujeres y un $50,82 \%$ de hombres, de los cuales, el $73,77 \%$ cursan o han cursado Educación Secundaria Obligatoria, el 13,11\% Bachillerato, el 9,84\% Formación Profesional Básica y el 3,28\% Educación Primaria. En relación a los adolescentes pertenecientes al centro de menores (ver Figura 2), el 51,72\% son mujeres y el 48,28\% hombres y, por su parte, han cursado o están cursando un 62,07\% Educación Secundaria Obligatoria, el 18,97\% Formación Profesional Básica, el 10,34\% Educación Primaria y el 8,62\% Formación Profesional Superior.

Figura 1. Relación de las edades con el sexo en jóvenes pertenecientes a centros normalizados

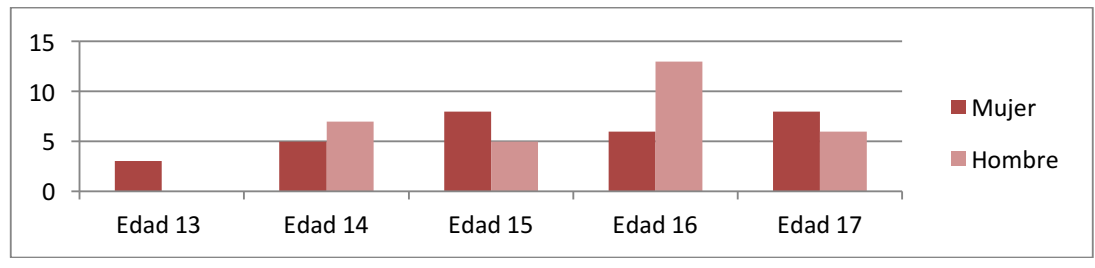

Figura 2. Relación de las edades con el sexo en jóvenes pertenecientes a un centro de menores

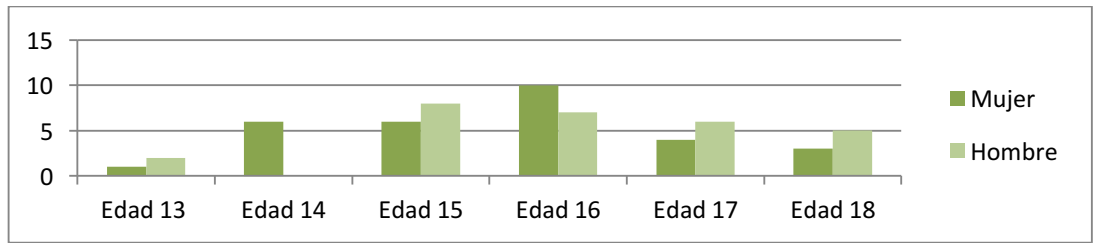

Respecto al estado sentimental, la distribución es la que se muestra en las Figuras 3 y 4 : 
Figura 3. Estado sentimental de los adolescentes pertenecientes a centros normalizados

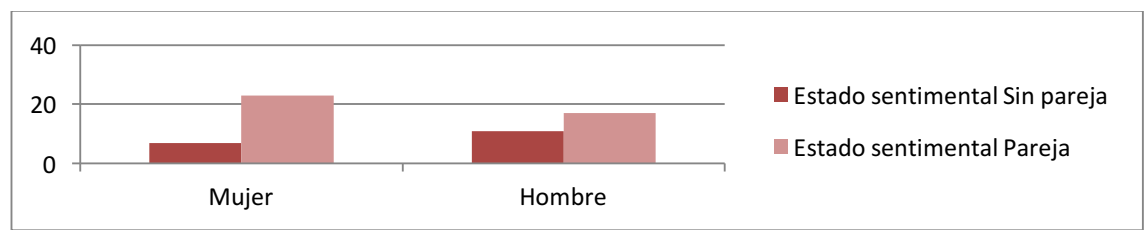

Figura 4. Estado sentimental de los adolescentes pertenecientes al centro de menores

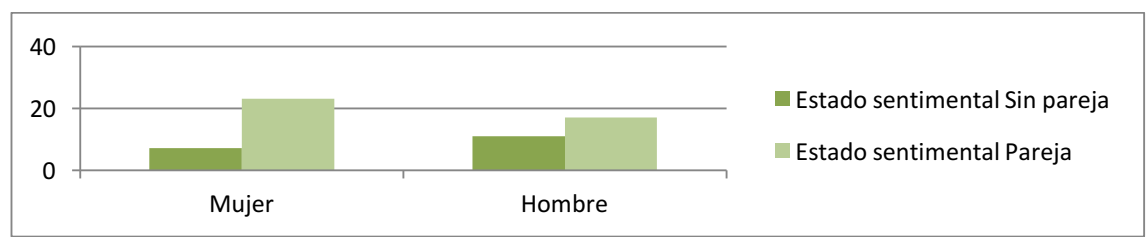

Con estos datos, podemos observar que la mayoría de los adolescentes encuestados tienen pareja estable, por lo que nos pueden proporcionar respuestas más realistas.

El primer bloque de preguntas del cuestionario está relacionado con la formación y el conocimiento que poseen sobre el concepto de violencia de género, sus tipos y los comportamientos esgrimidos.

Ante la pregunta “¿Has recibido en algún momento formación sobre violencia de género?”, las respuestas son las siguientes (ver Figuras 5 y 6 ):

Figura 5. Formación en V.G. de jóvenes pertenecientes a centros normalizados

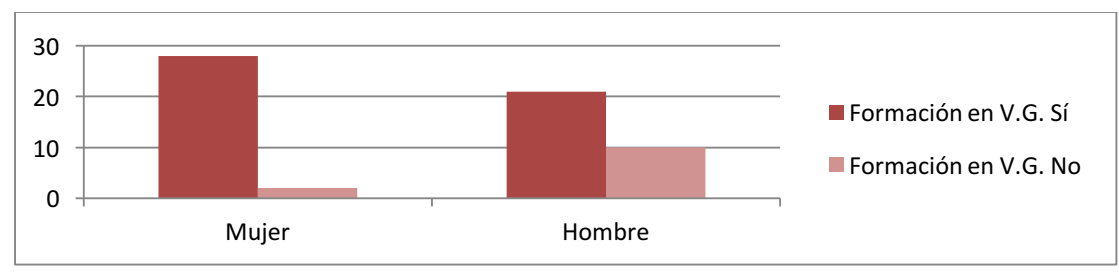

Figura 6. Formación en V. G. de jóvenes pertenecientes a un centro de menores

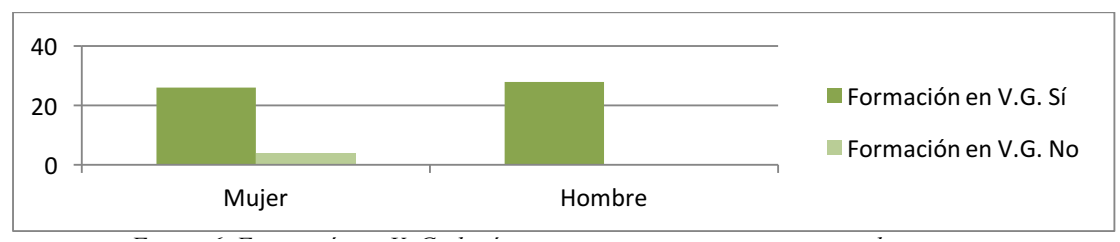

Según estos datos, observamos que la mayor parte de los jóvenes encuestados ha recibido, en algún momento, formación relacionada con este tema. Por lo que se deduce que en los diferentes centros se trabaja el tema de la violencia de género.

En relación a la pregunta “¿Pueden ser los hombres víctimas de violencia de género (VG)?” y la afirmación "La violencia de género está relacionada con..." responden (ver Figuras 7, 8, 9 y 10): 
Figura 7. Conocimiento de los jóvenes pertenecientes a centros normalizados sobre el concepto de V.G.

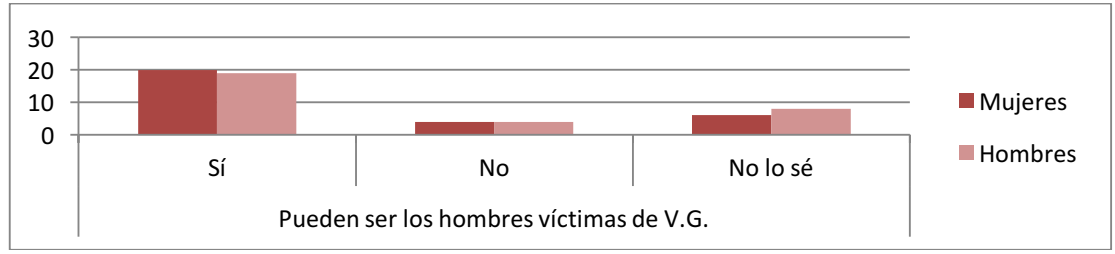

Figura 8. Conocimiento de los jóvenes pertenecientes a un centro de menores sobre el concepto de V.G.

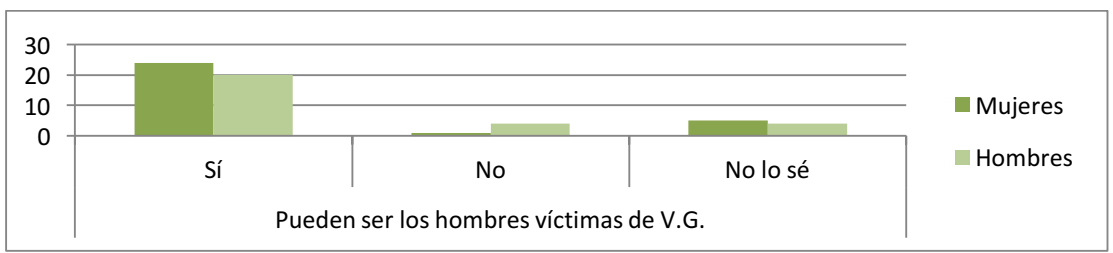

Figura 9. Tipos de V.G. existentes según jóvenes pertenecientes a centros normalizados

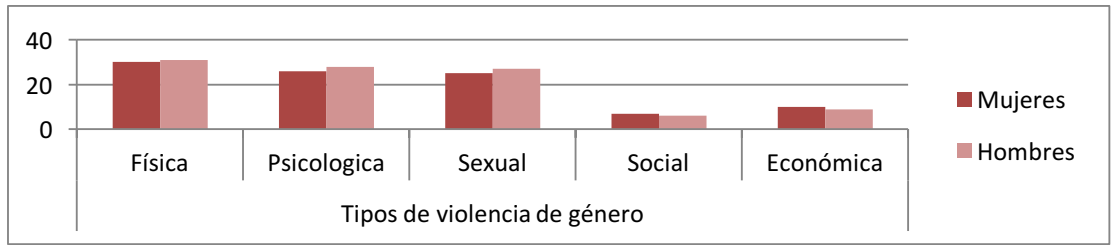

Figura 10. Tipos de V.G. existentes según jóvenes pertenecientes a un centro de menores

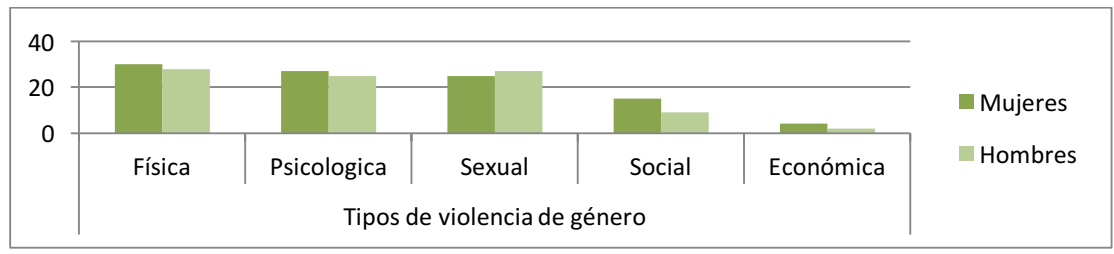

A la luz de estos resultados podemos concluir que, tanto unos jóvenes como otros, no tienen claro el verdadero concepto de violencia de género, ya que consideran que tanto hombres como mujeres pueden ser víctimas de este maltrato.

Por otro lado, en relación con los tipos de violencia, ambos grupos tienen claro que la violencia física, psicológica y sexual son tipos de violencia de género, pero solo una minoría sabe que la violencia económica también es un tipo de violencia de género.

A las preguntas "¿Cuándo crees que comienzan las primeras agresiones en la pareja?, “¿Conoces algún caso cercano donde se haya producido violencia de género?” y “¿Qué harías si vieses algún acto de violencia de género?" responden (ver Figuras 11, 12, 13, 14, 15 y 16): 
Figura 11. Comienzo de las agresiones en la pareja según jóvenes pertenecientes a un centro normalizado

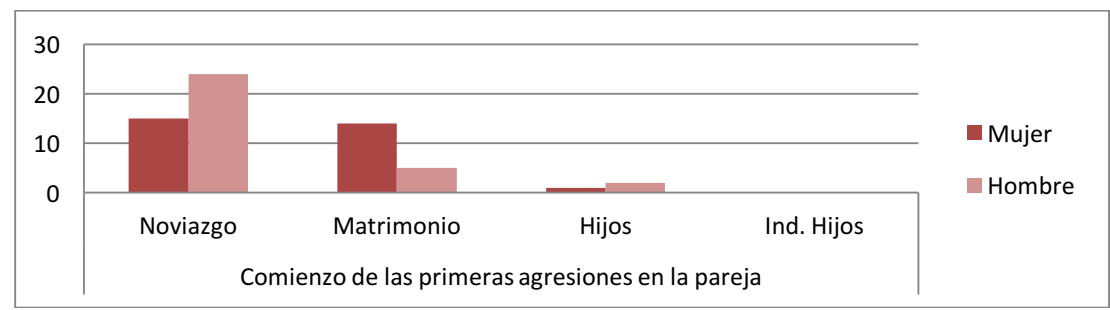

Figura 12. Comienzo de las agresiones en la pareja según jóvenes pertenecientes a centro de menores

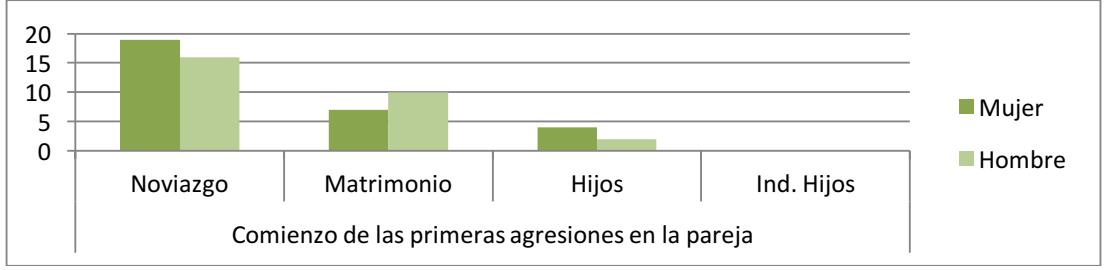

Figura 13. Conocimiento de jóvenes pertenecientes a centros normalizados de casos de V.G.

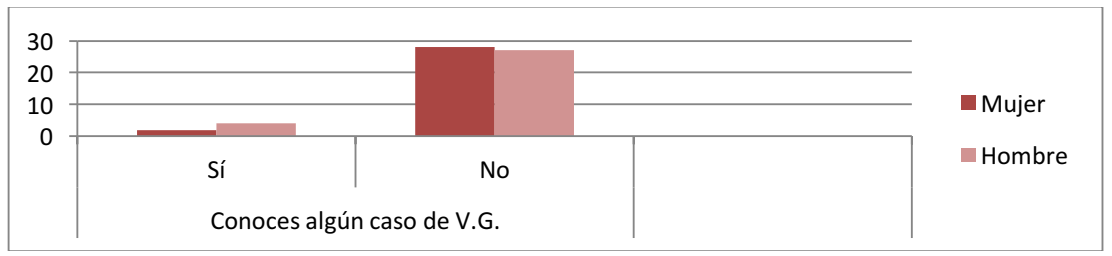

Figura 14. Conocimiento de jóvenes pertenecientes a un centro de menores de casos de V.G.

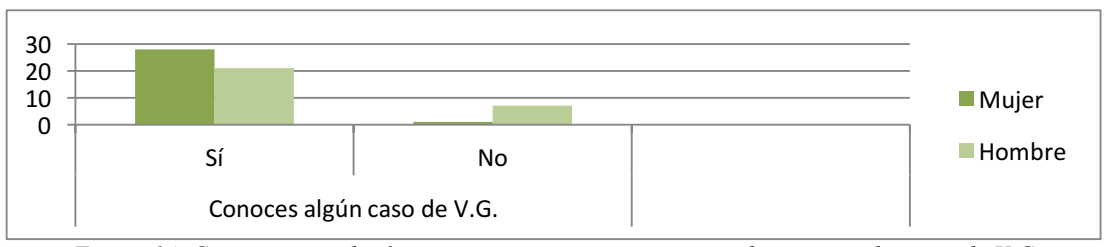

Figura 15. Actuación de los jóvenes pertenecientes a centros normalizados ante casos de V.G.

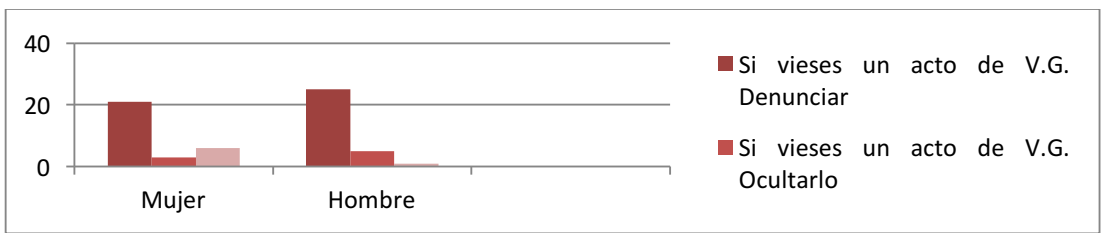


Figura 16. Actuación de los jóvenes pertenecientes a un centro de menores ante casos de V.G.

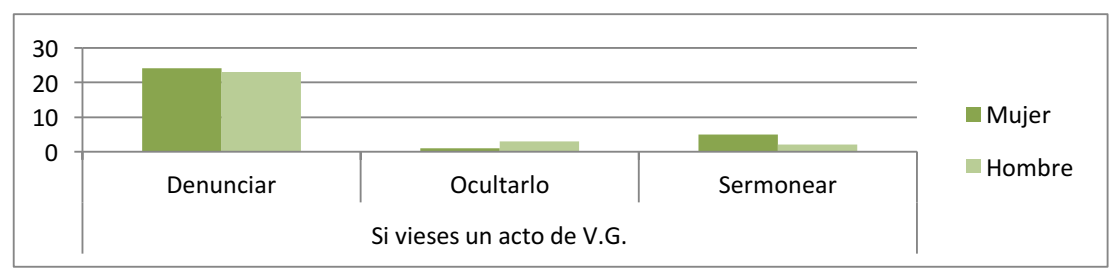

Tras analizar estas respuestas, se concluye que ambos grupos consideran de forma mayoritaria que la violencia de género puede aparecer ya en el noviazgo. También coinciden en que denunciarían al agresor si viesen un caso de violencia de género.

Cabe destacar que en relación a si conocen algún caso de violencia de género, las respuestas de ambos grupos son diferentes, ya que los jóvenes de centros normalizados responden en su mayoría que no y los pertenecientes al centro de menores contestan afirmativamente.

A las afirmaciones "Un hombre trata mal a su pareja porque ésta hace algo que no debe", "Muchas chicas van provocando y por eso sus parejas son violentas con ellas" y "Si una chica no se arregla para su chico, es normal que él se busque a otra", las respuestas son las siguientes (ver Figuras 17, 18, 19, 20, 21 y 22):

Figura 17. Visión de los jóvenes pertenecientes a centros normalizados sobre la culpabilidad de la víctima

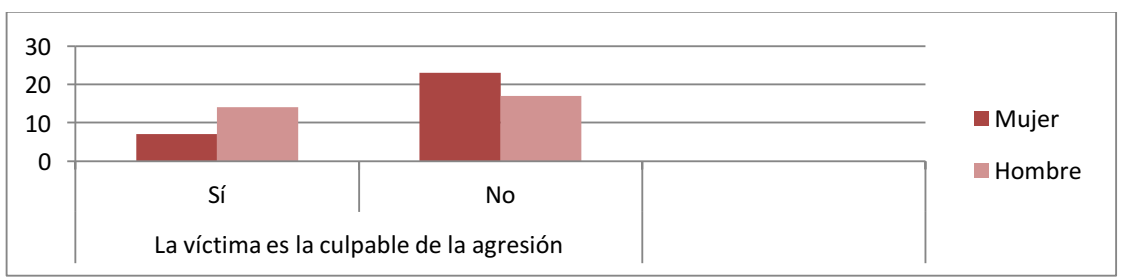

Figura 18. Visión de los jóvenes pertenecientes a un centro de menores sobre la culpabilidad de la víctima

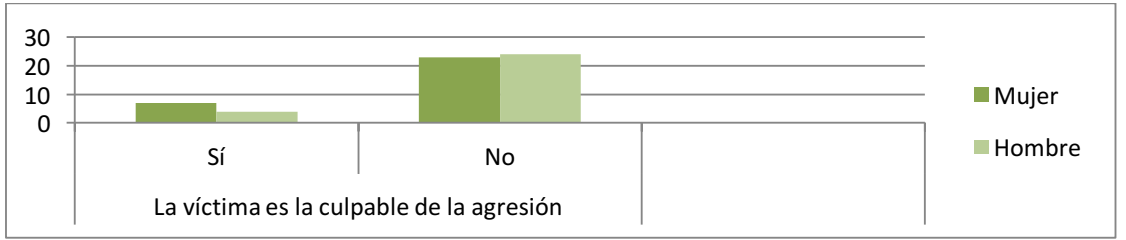

Figura 19. Visión de jóvenes pertenecientes a centros normalizados ante la "provocación" de las mujeres

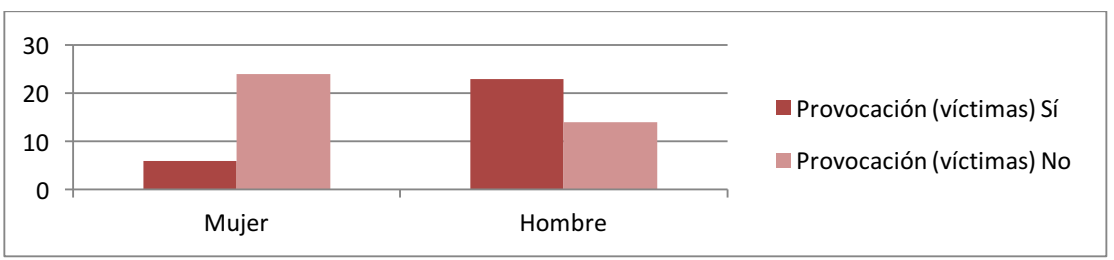


Figura 20. Visión de jóvenes pertenecientes a centros de menores ante la "provocación" de las mujeres

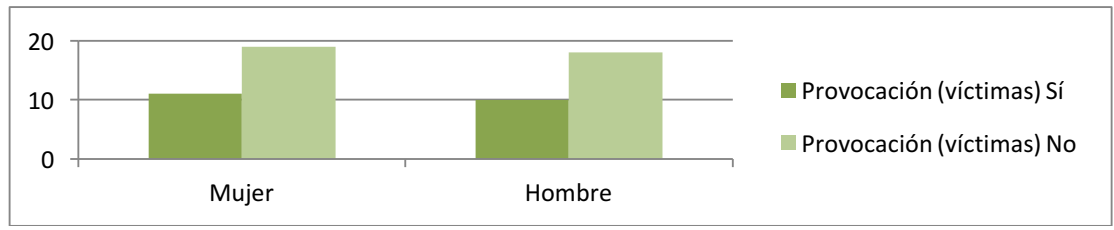

Figura 21. Visión de los jóvenes pertenecientes a centros normalizados ante el cambio de pareja de los agresores

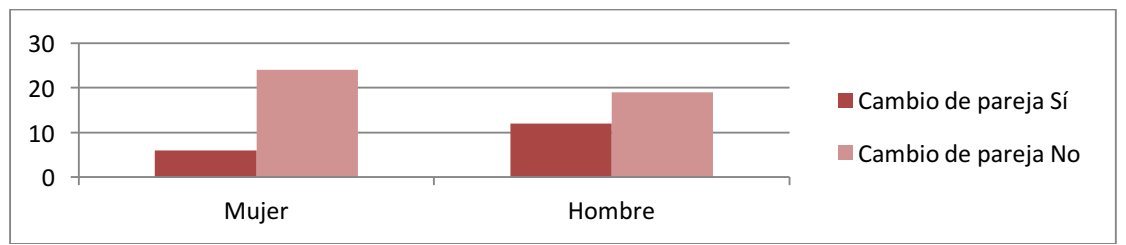

Figura 22. Visión de los jóvenes pertenecientes a centros de menores ante el cambio de pareja de los agresores

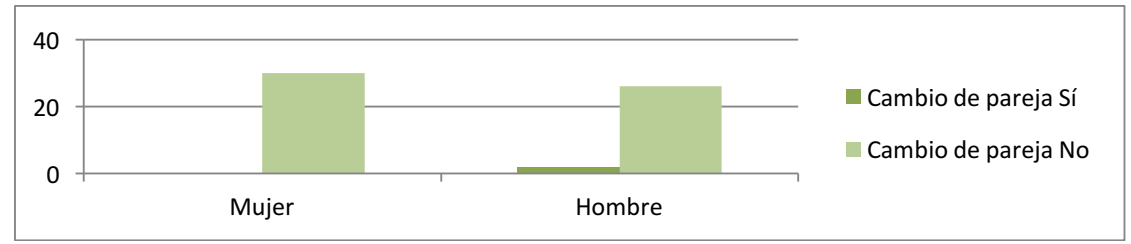

Después de analizar las respuestas, podemos destacar que la mayoría han respondido a estas afirmaciones de manera negativa y no se observan diferencias significativas entre un grupo y otro.

Las siguientes cuestiones forman parte del segundo bloque, relacionado con los mitos y las falsas creencias.

A la afirmación "Los maltratadores son personas con poca cultura", ambos grupos responde de la siguiente manera (ver Figura 23 y 24 ):

Figura 23. Visión de los jóvenes pertenecientes a centros normalizados sobre el mito de la poca cultura

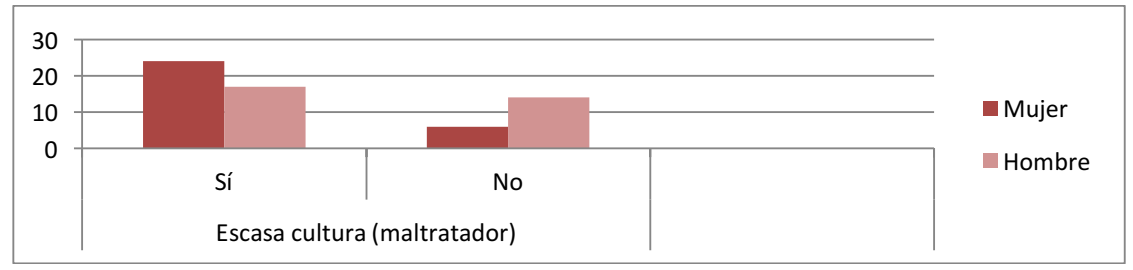


Figura 24. Visión de los jóvenes pertenecientes a centros de menores sobre el mito de la poca cultura

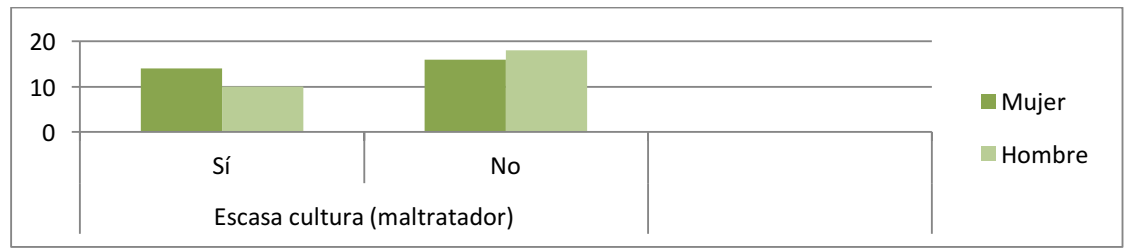

Después de observar los datos obtenidos en ambos grupos, se advierte que existen diferencias a la hora considerar la escasa cultura de los agresores como factor que influye a la hora de ejercer violencia de género. En tal sentido, se detecta que los jóvenes normalizados han interiorizado este mito.

A la afirmación "Los maltratadores tienen algún tipo de enfermedad mental" contestan en el siguiente sentido (ver Figura 25 y 26 ):

Figura 25. Visión de los jóvenes pertenecientes a centros normalizados ante el mito de la enfermedad mental

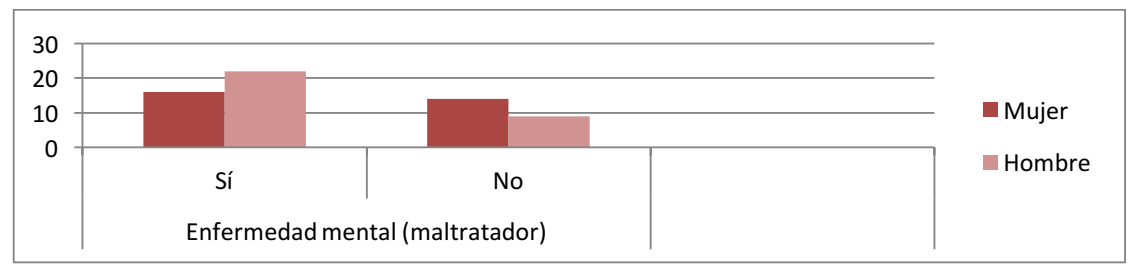

Figura 26. Visión de los jóvenes pertenecientes a un centro de menores ante el mito de la enfermedad mental

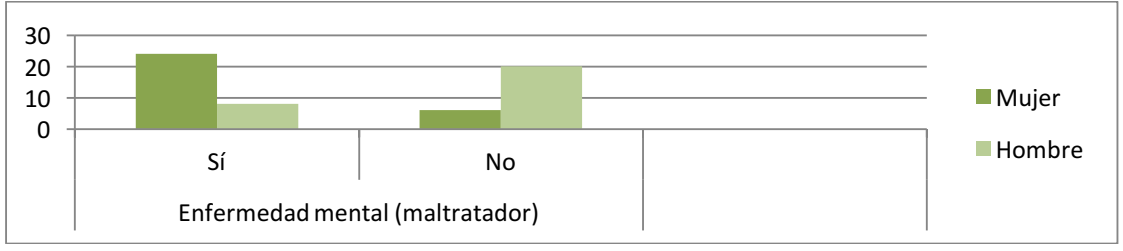

Lo que observamos en este ítem es que ambos grupos coinciden en las respuestas afirmativas ante este mito.

A la afirmación "La mayoría de mujeres víctimas de violencia de género en España son inmigrantes" (ver Figuras 27 y 28), responden: 
Figura 27. Visión de los jóvenes pertenecientes a centros normalizados ante el mito de la víctima inmigrante

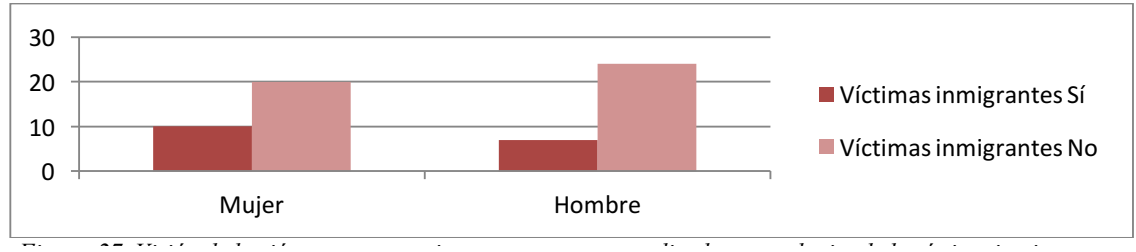

Figura 28. Visión de los jóvenes pertenecientes a un centro de menores ante el mito de la víctima inmigrante

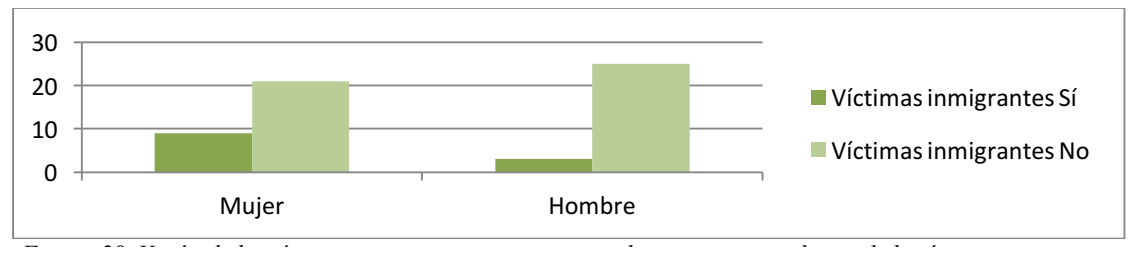

Se observa que en esta cuestión los menores coinciden en sus respuestas.

A la afirmación "Hay pocos asesinatos por violencia de género", sus respuestas son (ver Figuras 29 y 30$)$ :

Figura 29. Visión de los jóvenes pertenecientes a centros normalizados ante los asesinatos por V.G.

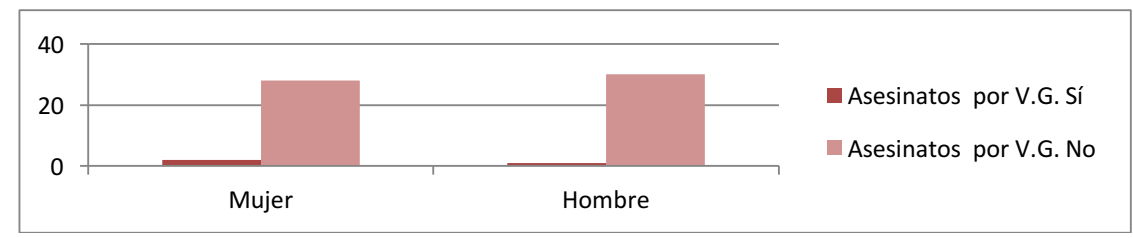

Figura 30. Visión de los jóvenes pertenecientes a un centro de menores ante los asesinatos por V.G.

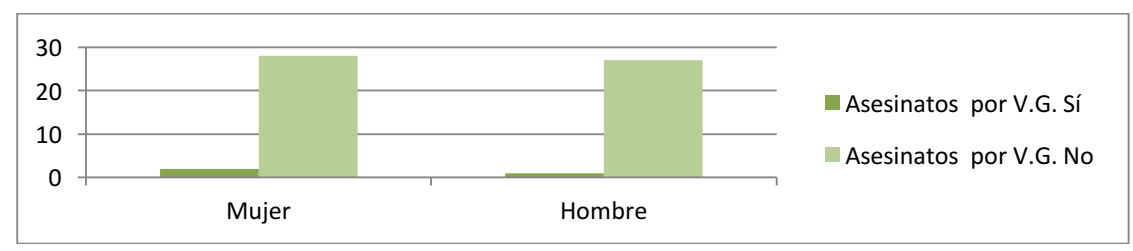

Ambos grupos consideran, mayoritariamente, que hay demasiados asesinatos por este tipo de violencia.

A la afirmación "Las mujeres maltratadas suelen tener atracción hacia hombres agresivos" (ver Figuras 31 y 32$)$, responden: 
Figura 31. Visión de los jóvenes pertenecientes a centros normalizados ante el mito de la atracción hacia hombres agresivos

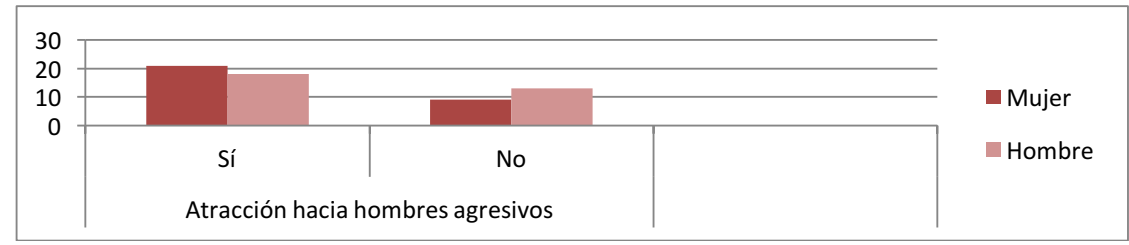

Figura 32. Visión de los jóvenes pertenecientes a un centro de menores ante el mito de la atracción hacia hombres agresivos

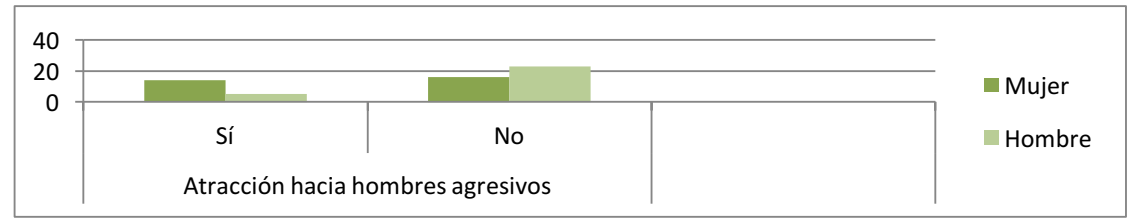

Existen grandes diferencias entre ambos grupos, por un lado los jóvenes normalizados consideran este tipo de mito real y, por otro, los adolescentes pertenecientes al centro de menores responden a esta pregunta de forma negativa.

A la afirmación "Es normal tener celos cuando estas enamorado/a, sus respuestas son (ver Figuras 33 y 34$) ":$

Figura 33. Visión de los jóvenes pertenecientes a centros normalizados sobre los celos

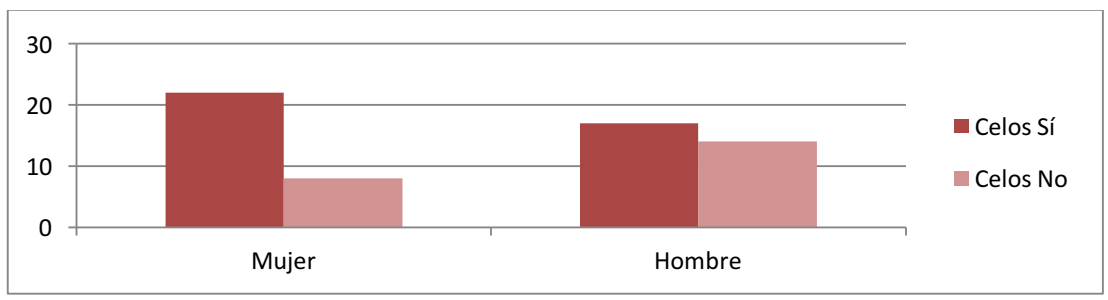

Figura 34. Visión de los jóvenes pertenecientes a un centro de menores sobre los celos

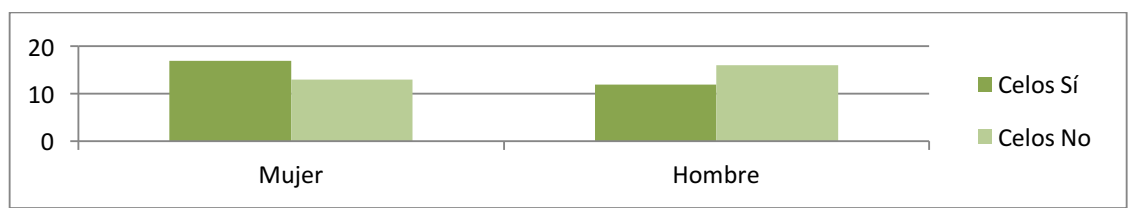

Destaca sobremanera que ambos grupos consideran los celos como un elemento importante dentro de las relaciones de pareja, aunque en el grupo perteneciente al centro de menores globalmente ha habido el mismo número de respuestas afirmativas que negativas.

A las afirmaciones "Solo existe un amor verdadero, Ios demás son solo pasajeros" y "Todas las 
personas tenemos nuestra media naranja, solo hay que encontrarla" responden (ver Figuras 35, 36, 37 y 38$)$ :

Figura 35. Visión de los jóvenes pertenecientes a centros normalizados sobre el mito del amor verdadero

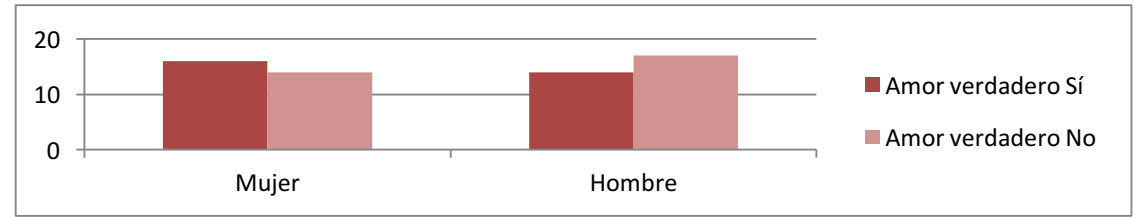

Figura 36. Visión de los jóvenes pertenecientes a un centro de menores sobre el mito del amor verdadero

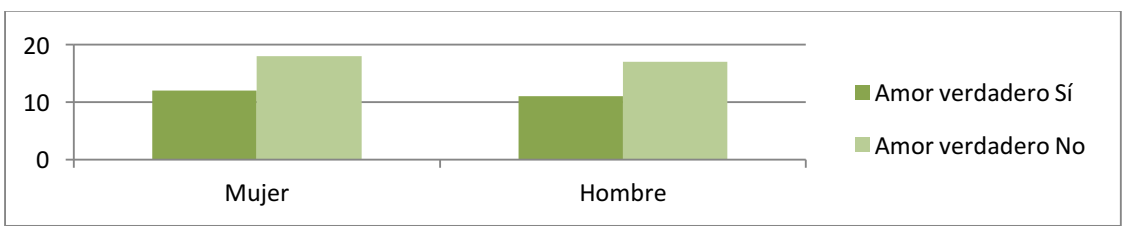

Figura 37. Visión de los jóvenes pertenecientes a centros normalizados sobre el mito de la media naranja

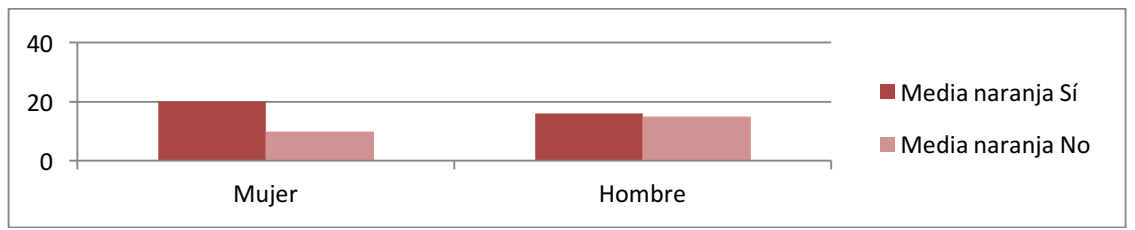

Figura 38. Visión de los jóvenes pertenecientes a un centro de menores sobre el mito de la media naranja

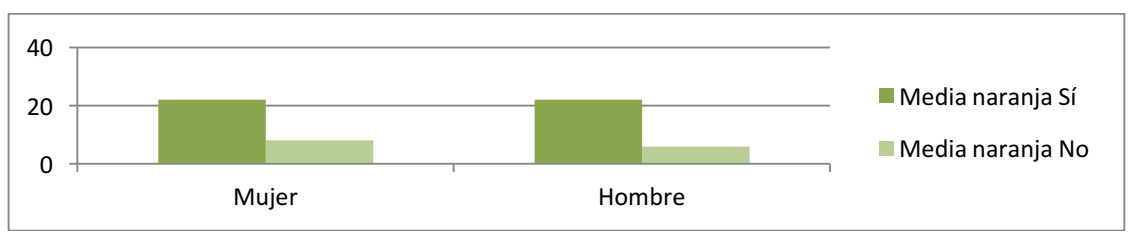

Se detecta que ambos grupos responden negativamente ante el mito del amor verdadero, pero al preguntarles sobre el mito de la media naranja responden de manera afirmativa por lo que ambos grupos creen en él.

A la afirmación "Cuando tienes pareja debes pasar la gran parte de tu tiempo con ella", responden (ver Figuras 39 y 40):

Figura 39. Visión de los jóvenes pertenecientes a centros normalizados sobre el tiempo en pareja

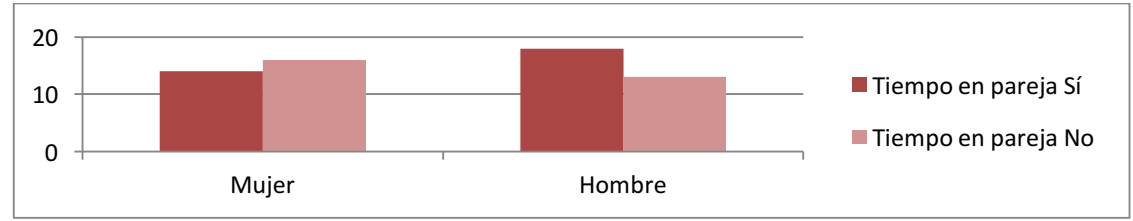


Figura 40. Visión de los jóvenes pertenecientes a un centro de menores sobre el tiempo en pareja

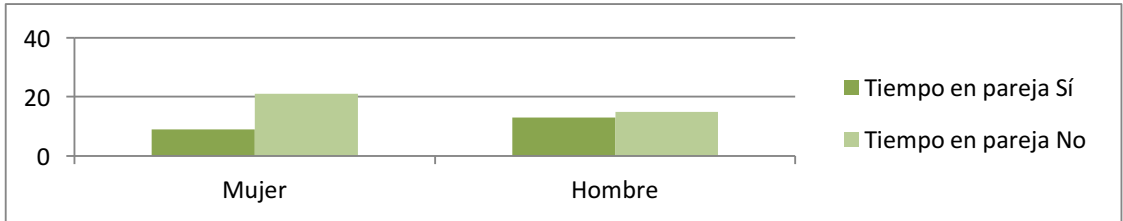

Según las respuestas obtenidas, los jóvenes de centros normalizados consideran que deben pasar la mayor parte de su tiempo con su pareja, mientras que los adolescentes del centro de menores consideran que esto no es así.

A la afirmación "Cuando estoy con una pareja me gusta que los/as demás chicos/as lo/a miren" contestan (ver Figuras 41 y 42):

Figura 41. Visión de los jóvenes pertenecientes a centros normalizados sobre la mirada de otras personas a su pareja

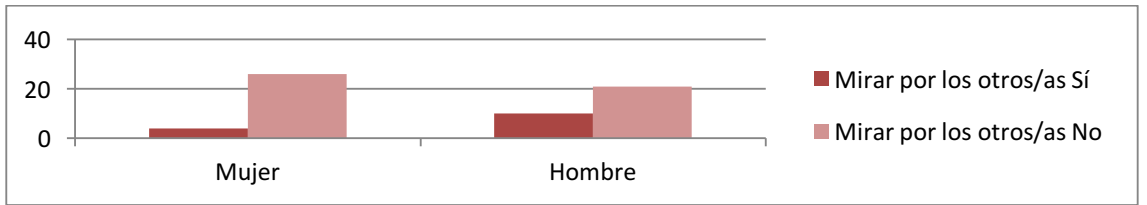

Figura 42. Visión de los jóvenes pertenecientes a un centro de menores sobre la mirada de otras personas a su pareja

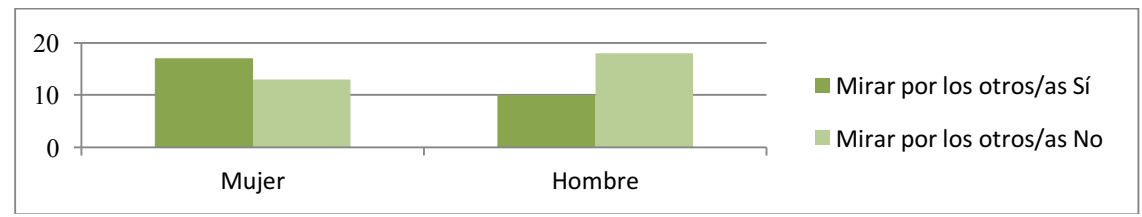

Con respecto a esta afirmación, ambos grupos coinciden en que no les gusta que otras personas miren a sus parejas, lo que puede conllevar asociado desconfianza y celos en la pareja.

Finalmente, el tercer bloque de preguntas está relacionado con los medios de comunicación de masas y las nuevas tecnologías.

A la pregunta "Los medios de comunicación (cine, televisión...), ¿crees que influyen en nuestra manera de pensar? responden (ver Figuras 43 y 44 ):

Figura 43. Visión de los jóvenes pertenecientes a centros normalizados sobre la influencia de los medios de comunicación

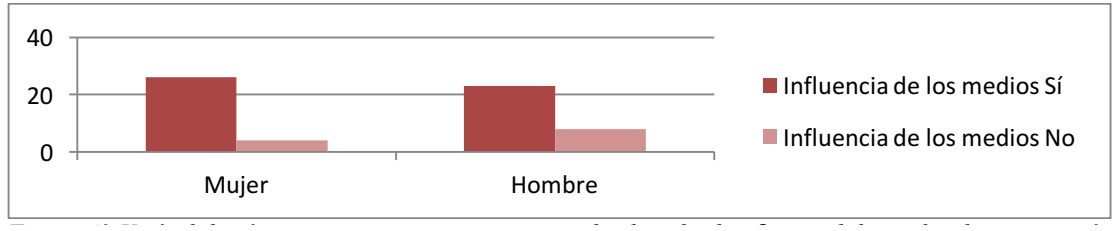


Figura 44. Visión de los jóvenes pertenecientes a un centro de menores sobre la influencia de los medios de comunicación

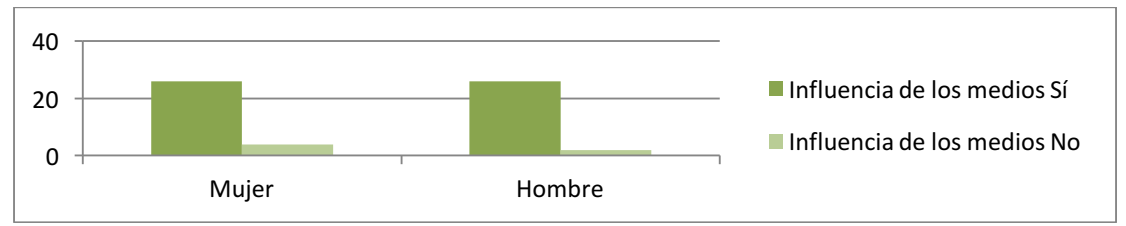

A este respecto, los ambos grupos encuestados han respondido mayoritariamente de manera afirmativa, por lo que son conscientes de la gran importancia de éstos.

A la afirmación "Cuando estoy en internet (redes sociales), le doy mucha importancia a las fotos que subo y actualizo con frecuencia mi perfil" contestan (ver Figuras 45 y 46):

Figura 45. Visión de los jóvenes pertenecientes a centros normalizados sobre las redes sociales

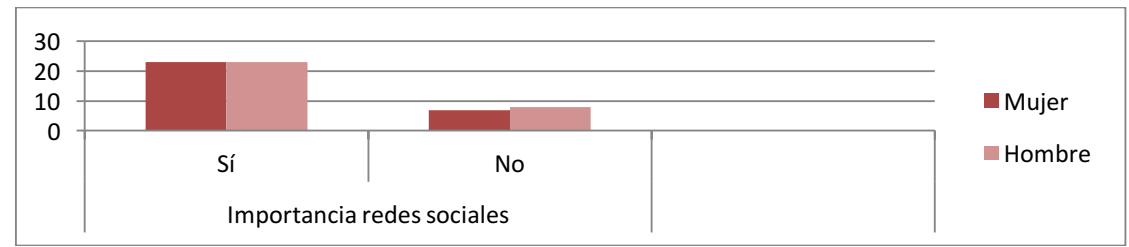

Figura 46. Visión de los jóvenes pertenecientes a un centro de menores sobre las redes sociales

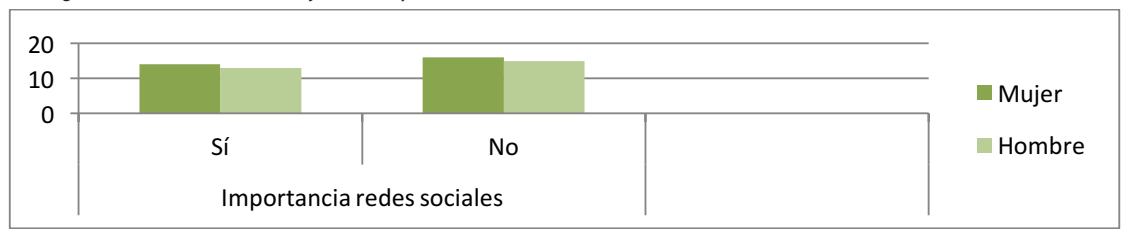

A este respecto, podemos afirmar que los jóvenes de centros normalizados le dan más importancia y utilizan más las redes sociales que los adolescentes del centro de menores.

A la afirmación "Me gusta tener muchos amigos en las redes sociales, no importa si no los conozco en persona" responden (ver Figuras 47 y 48):

Figura 47. Visión de los jóvenes pertenecientes a centros normalizados sobre las amistades desconocidas en las redes sociales

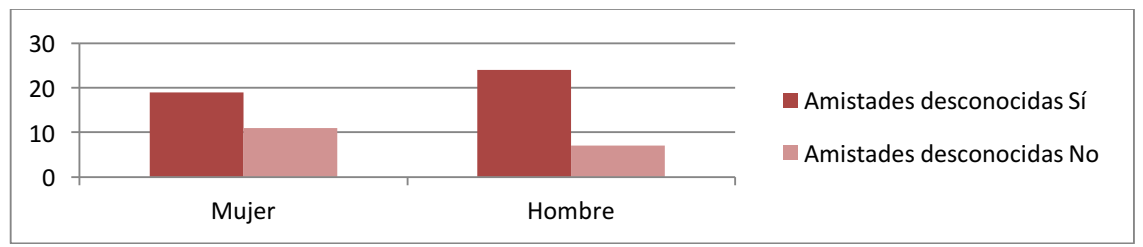


Figura 48. Visión de los jóvenes pertenecientes a un centro de menores sobre las amistades desconocidas en las redes sociales

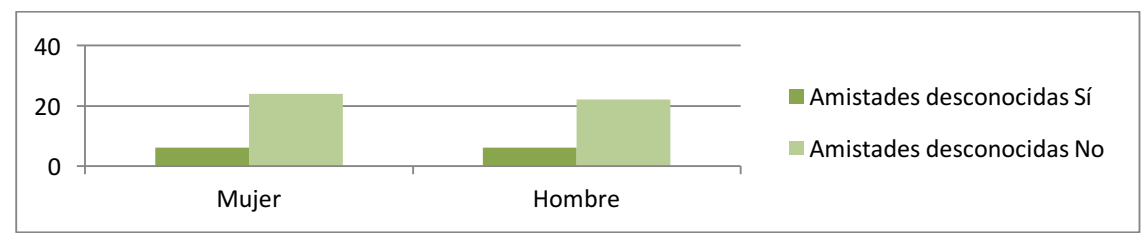

Tras las respuestas obtenidas en esta afirmación, se puede observar cómo a los jóvenes pertenecientes a centros normalizados no les importa agregar desconocidos a sus redes sociales, mientras que los adolescentes pertenecientes al centro de menores consideran lo contrario.

A la frase "Si un/a chico/a me habla mucho por las redes sociales e intenta quedar conmigo sin que yo quiera" señalan que (ver Figuras 49 y 50 ):

Figura 49. Visión de los jóvenes pertenecientes a centros normalizados sobre el contacto insistente en las redes sociales

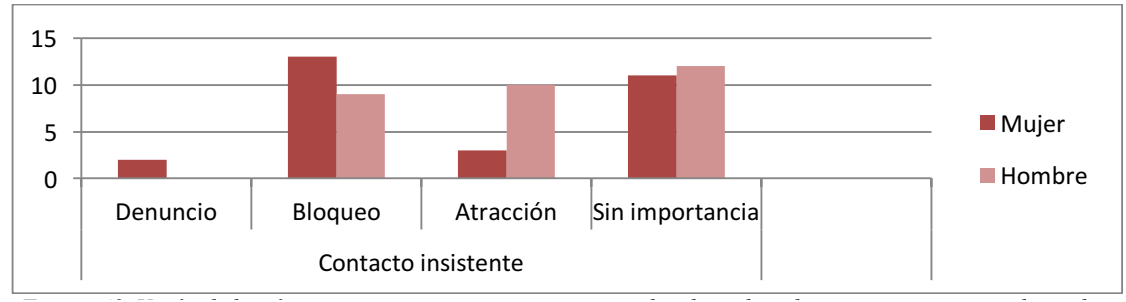

Figura 50. Visión de los jóvenes pertenecientes a un centro de menores sobre el contacto insistente en las redes sociales

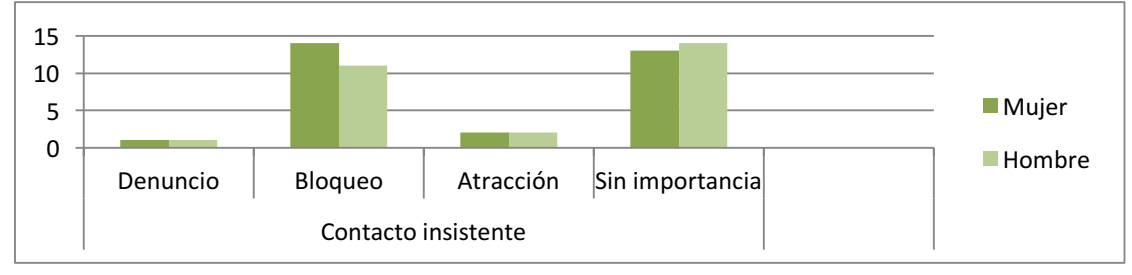

En relación a esta pregunta ambos grupos de adolescentes coinciden, en gran parte, en que no le darían importancia a esta situación 0 , en segundo lugar, que bloquearían a dicha persona.

Finalmente, a la frase "Si envío un WhatsApp a mi pareja..." las respuestas sobre el momento en que pretende que el otro le conteste son (ver Figuras 51 y 52 ): 
Figura 51. Visión de los jóvenes pertenecientes a centros normalizados sobre el control en WhatsApp

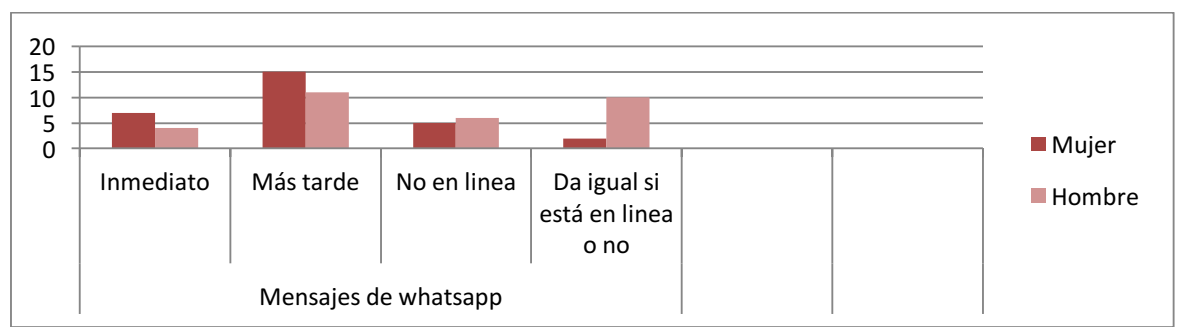

Figura 52. Visión de los jóvenes pertenecientes a un centro de menores sobre el control en WhatsApp

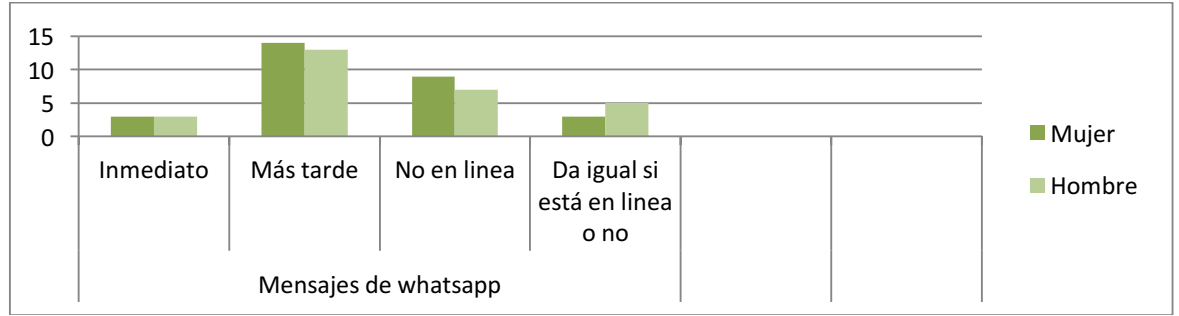

Después de analizar estos datos, se observa que no existen diferencias entre ambos grupos, destacando como respuesta mayoritaria "no me importa que me contesten más tarde", seguido -en los jóvenes de centros normalizados- de la respuesta "me gusta que me conteste enseguida, nunca me fijo si está en línea o no". Por el contrario, en los jóvenes pertenecientes al centro de menores, la segunda respuesta más elegida es "no me importa que me conteste más tarde, siempre y cuando no esté en línea".

\section{CONCLUSIONES}

Después de analizar la información obtenida se observa que se ha respondido a los dos objetivos planteados en el presente trabajo.

En relación al primer objetivo general planteado. Se han estudiado los diferentes estereotipos y falsas creencias imperantes debido a la sociedad androcéntrica en la que nos encontramos, el conocimiento sobre el concepto y tipos de violencia de género, se han analizado las conductas tolerantes y de rechazo ante esta situación y por último, la consciencia de los jóvenes en las redes sociales y la importancia de los medios de comunicación.

Con respecto al segundo objetivo general planteado en el presente estudio, se han observado las posibles diferencias existentes entre el grupo perteneciente a un centro de menores y el grupo perteneciente a diferentes centros normalizados, con el objetivo de estudiar las diferencias en el aprendizaje de roles, mitos y falsas creencias, conceptos sobre este tipo de violencia y las actitudes que toman ante comportamientos violentos.

En relación con los conocimientos que poseen acerca del concepto y tipos de violencia de género, así como las actitudes ante estos comportamientos no se observan diferencias significativas entre ambos grupos. Sin embargo, en relación al conocimiento de algún caso de éste tipo de violencia, sí aparecen diferencias, puesto que los pertenecientes al centro de menores sí afirman conocer casos. 
En cuanto a las actitudes de rechazo o tolerantes ante situaciones de maltrato, no se aprecian grandes diferencias en las respuestas emitidas por ambos grupos de jóvenes. Todos los adolescentes han respondido con actitudes de rechazo ante las situaciones planteadas. Se puede considerar que la gran mayoría de los jóvenes encuestados están concienciados de actitudes machistas presentes en nuestra sociedad.

Respecto a los mitos y falsas creencias, el estudio revela que los jóvenes pertenecientes al centro de menores parecen más conscientes de que las preguntas planteadas son mitos. Cabe desta-

car las preguntas sobre los mitos del amor verdadero y la media naranja, en la primera afirmación ambos grupos de jóvenes han respondido que esta afirmación es un mito, pero al preguntárselo asociándolo al mito de la media naranja, la mayor parte de los adolescentes han respondido de manera afirmativa, por ello, consideramos que ambos jóvenes siguen creyendo en estos mitos, y en consecuencia, buscan a la pareja 'ideal'.

Continuando con los datos obtenidos en relación al uso 0 abuso de los jóvenes de las redes sociales y medios de comunicación, se observa que aunque, ambos grupos son conscientes de la influencia de los medios en nuestra vida diaria, los jóvenes de centros normalizados tienen mayor dependencia de las redes sociales, destacando la escasa importancia que ambos grupos le otorgan al acoso potencial que pueden sufrir en las redes.

\section{REFERENCIAS BIBLIOGRÁFICAS}

Cantera, I., Estébanez, I. y Vázquez, N. (2009). Violencia contra las mujeres jóvenes, la violencia psicológica en las relaciones de noviazgo. Servicio de la Mujer del Módulo Psicosocial de DeustoSan Ignacio. Gobierno Vasco.

González, R. y Santana, J.D. (2001). La violencia en parejas jóvenes. Psicothema, 13(1), 127-131. Recuperado de:

https://www.unioviedo.es/reunido/index.php/PST/article/view/7856/7720

Organización Mundial de la Salud (2017). Desarrollo en la adolescencia. Recuperado de: http://www.who.int/maternal_child_adolescent/topics/adolescence/dev/es/

Organización de las Naciones Unidas (1994). Asamblea General: Declaración sobre la eliminación de la violencia contra la mujer. Recuperado de:

http://www.un.org/es/comun/docs/?symbol=A/RES/48/104\&Lang=S

Williams, J.E. y Best, D.L. (1990). Measuring sex stereotipes: A multinational study. Newbury Park, California: Sage. 